\title{
Linking Microbial Community Profiles to Subsurface Zonation by Geochemical Properties
}

\author{
HAN-SUK KIM ${ }^{1}$, Ho YOUNG JO ${ }^{1,3}$, YONG-SUNG JOO², \\ AND MAN JAE KWON*1,3 \\ ${ }^{1}$ SMART-SEM Research Center, Korea University, \\ Seoul, Korea, (num177@korea.ac.kr) \\ ${ }^{2}$ Department of Statistics, Dongguk University, Seoul, \\ Korea \\ ${ }^{3}$ Department of Earth and Environmental Sciences, \\ Korea University, Seoul, Korea \\ (*correspondence: manjaekwon@korea.ac.kr )
}

Distinct microbial community diversity and structure has been observed in various Earth's critical zones (i.e., nearsurface environents) with soil depth. Here we investigated microbial community compositions and geochemistical properites along a soil vertical profile spanning 0 to $\sim 50 \mathrm{~m}$ depths including top soil, vadose zone, capillary fringe and saturated zones in a testbed site formerly used as a famland for several decades. Based on the results of 16S-rRNA microbial community analyses, microbial OTU richness showed unique patterns along the soil vertical profile. The microbial diversity was highest in the top soil layer and was also significantly higher in the capillary fringe than in unsaturated and saturated zones. At the phylum level, Proteobacteria, Actinobacteria, Acidobacteria and Chloroflexi were more abundant in the top soil layer, while Bacteroidetes, whereas Firmicutes, Actinobacteria and Proteobacteria showed relatively high abundance in the capillary fringe zone. Redundancy correlation analyses (RDA) showed that the community in the soil samples was significantly affected by $\mathrm{NO}_{3}{ }^{-}$and $\mathrm{SO}_{4}{ }^{2-}$ in the unsaturated zone; chromium, bioavailable iron and nickel in the capillary fringe; vandium in the saturated zone $(\mathrm{P}<0.05$, respectively). Microbial numbers and community compositions were also significantly different in between soil and groundwater samples likely because microorganisms attached to the solid particles might better access to nutrients than those suspended in aquifer. The abundance of Alphaproteobacteria (Reyranellales, Sphingomonadales), Betaproteobacteria (Rhodocyclaceae, Gallionellaceae, Sulfuricellaceae) and Gammaproteobacteria (Xanthomonadales) was much higher in groundwater samples compared to in soil samples. Microbial numbers in soil samples by Most Probable Numbers were $30 \sim 400$ times higher than those in groundwater samples. This study will discuss potential roles of microbial community structure and functional diversity on geochemical properties in each critical zone. 